\title{
A study of the TP53 Germline Mutation and Clinicopathologic Features in Thai Children with Adrenocortical Carcinoma
}

\author{
Nadvadee Aungkawattanapong, M.D. ${ }^{1}$, Piti Techavichit, M.D. ${ }^{1}$, Supanun Lauhasurayotin, M.D. , \\ Kanhathai Chiengthong, M.D. ${ }^{1}$, Darintr Sosothikul, M.D. ${ }^{1}$ Chalinee Monsereenusorn, M.D. ${ }^{2}$, \\ Vichit Supornsilchai, M.D., Ph.D. ${ }^{1}$, Kanya Suphapeetiporn, M.D., Ph.D. ${ }^{1}$, \\ Chinachote Teerapakpinyo, Ph.D. ${ }^{3}$, Shanop Shuangshoti, M.D. ${ }^{4}$
}

${ }^{1}$ Clinical Research for Holistic Management in Pediatric Hematological and Oncology, Department of Pediatrics, Faculty of Medicine, King Chulalongkorn Memorial Hospital, Chulalongkorn University, Pathumwan, Bangkok 10330, Thailand. ${ }^{2}$ Division of Hematology/Oncology, Department of Pediatrics, Phramongkutklao Hospital and Phramongkutklao College of Medicine, Ratchatewi, Bangkok 10400, Thailand

${ }^{3}$ Chula GenePro Center, Research Affairs, Faculty of Medicine, Chulalongkorn University, Pathumwan, Bangkok 10330, Thailand. ${ }^{4}$ Department of Pathology, Faculty of Medicine, King Chulalongkorn Memorial Hospital, Chulalongkorn University, Pathumwan, Bangkok 10330, Thailand.

Received 12 October 2020 • Revised 21 December 2020 • Accepted 28 December 2020 • Published online 11 May 2021

\section{Abstract:}

Objective: To determine the clinicopathologic features and germline tumor protein p53 (TP53) mutation in children with adrenocortical carcinoma (ACC).

Material and Methods: This was a retrospective study. From 2009 to 2018, children with ACC from King Chulalongkorn Memorial Hospital and Phramongkutklao Hospital were enrolled into the study. Clinical presentations and hormonal profiles were recorded. Mutation analyses of the TP53 gene were acquired using the next-generation-sequencing method which was performed for germline samples of all patients and the parents of those who tested positive.

Result: Two males and six females with ACC were enrolled into this study. The median age at diagnosis was 25.5 months (range 10 to 67 months). All participants had virilization, either virilization only $(n=3)$ or associated with Cushing $(n=5)$. All participants had had surgery to completely remove all of the tumor and three participants had received adjuvant chemotherapy consisting of etoposide, doxorubicin, and cyclophosphamide. All participants had three different

Contact: Piti Techavichit, M.D.

Department of Pediatrics, Faculty of Medicine, King Chulalongkorn Memorial Hospital,

Chulalongkorn University, Pathumwan, Bangkok 10330, Thailand.

E-mail: Piti.T@chula.ac.th

This is an open access article under the CC BY-NC-ND license

(http://www.jhsmr.org/index.php/jhsmr/about/editorialPolicies\#openAccessPolicy).

J Health Sci Med Res 2021;39(6):491-502 doi: $10.31584 / j h s m r .2021810$ www.jhsmr.org 
known mutations in the TP53 gene: c.1010G>A (p.R337H), c.916C>T (p.R306*) and c.743G>A (p.R248Q). Two of the three participants with TP53 mutations had pulmonary metastasis. One participant had wild-type TP53 and pulmonary recurrence occurred one year after diagnosis. The median follow-up time was 31 months (range 10 to 168 months. As of this writing, seven participants survived without evidence of recurrence. No second malignancy was found in all participants. One participant who had c.916C>T died of pulmonary metastasis 10 months after diagnosis.

Conclusion: We successfully identified three different germline TP53 mutations in patients with ACC. Progression to pulmonary metastasis and recurrence were higher among participants with TP53 mutations. The treatment outcome of childhood ACC was good when surgery and adjuvant chemotherapy were used.

Keywords: Adrenocortical carcinoma, children, Germline mutation, Hereditary, Li- Fraumeni syndrome, TP53

\section{Introduction}

Adrenocortical carcinoma (ACC) is a rare adrenal gland tumor. In the United States of America (USA), the annual incidence of ACC is $0.2-0.3$ cases per million per year. ACC originates from the adrenal cortex which is responsible for the production of several hormones including mineralocorticoids, glucocorticoids and sex hormones. Thus, children with ACC can present with a Cushing appearance and symptoms of virilization or feminization as well as hypertension due to excess of mineralocorticoids. ${ }^{1,2}$ Comparing to adolescent and adult patients, Abiven et al. had reported that only $54.0 \%$ of patients (aged 11.2-88.6 years old) presented with endocrinological symptoms. In adults, only $76.0 \%$ of all adult patients with ACC had hormone-secreting tumors. ${ }^{3}$ The prognosis of ACC is good, especially among younger children. Mahendraraj and colleagues reported that the 5-year survival rates of patients younger than 5 years old, 5-9 years old and 1019 years old were $90.0 \%, 45.0 \%$ and $21.0 \%$, respectively. ${ }^{2}$ The incidence rate of ACC varies among different ethnic groups, indicating that genetics plays an important role in the development of ACC. One study reported that the incidence of ACC was higher in Southern Brazil than world-wide incidence, with an incidence rate of 3.4 cases per million per year. ${ }^{4}$ Some syndromes such as Beckwith-
Wiedemann syndrome, multiple endocrine neoplasia type I and Li-Fraumeni syndrome (the mutation of tumor protein p53 (TP53)) are important predisposing factors for ACC in children. ${ }^{5}$

TP53 is a tumor suppressor gene located on chromosome 17. Germline mutations of TP53 result in familial cancers, called Li-Fraumeni syndrome, which is inherited through an autosomal dominant pattern. ${ }^{5}$ Previous studies reported that the prevalence of this TP53 germline mutation in American and Canadian children with ACC ranged between $50.0-80.0 \%$, regardless of family history. ${ }^{6-8}$ Another study from Malaysia reported the prevalence of ACC in children was $75.0 \% .{ }^{9}$ One study reported that half of the patients with Li-Fraumeni syndrome had developed cancer by the age of 30 and $90.0 \%$ before the age of 60 years. $^{5}$ In contrast to adult-onset ACC, a study in Germany had reported that germline TP53 mutation was only $3.9 \%$ in patients with adult-onset ACC. ${ }^{10}$ Choong SS, et al. suggested using childhood ACC with germline TP53 mutation as a sentinel cancer for detecting families at risk of cancer. ${ }^{9}$ Treatments for patients with germline TP53 mutations should be done carefully; radiation therapy or some specific chemotherapy, to decrease the risk of a second malignancy in patients with TP53 mutations. ${ }^{11}$ 
To our knowledge, there are no studies to date on TP53 mutations in Thai children with ACC. This is the first study to assess germline TP53 mutations in Thai children with ACC. In this study we assess the frequency of germline TP53 mutations in Thai children with ACC and characterize the clinical features and outcomes of treatment.

\section{Material and Methods}

ACC patients aged 0 to 18 years with confirmed pathological report who attended King Chulalongkorn Memorial Hospital and Phramongkutklao Hospital between 2009-2018 were enrolled into the study. The study was approved by the Institutional Review Board of the Faculty of Medicine, Chulalongkorn University (Bangkok, Thailand; IRB.319/60). The study was conducted according to the Declaration of Helsinki. Assent was obtained from the children and written informed consent was obtained from a parent or legal guardian.

Clinical presentations, hormonal profiles and medical records were reviewed. Family pedigrees were obtained from thorough interviews with the parents.

TP53 sequencing was performed using the targeted next-generation-sequencing method at the Chula GenePRO Center Laboratory, Faculty of Medicine, Chulalongkorn University, Bangkok, Thailand.

All coding regions and canonical splice sites of the TP53 gene in the whole blood deoxyribonucleic acid (DNA) of the participants and parents were sequenced using next generation sequencing (the Illumina MiSeq System; Illumina). Enrichment of target genes was performed using GeneRead DNAseq Human Clinically Relevant Tumor Panel v2 according to the manufacturer's instructions (Catalog no. NGHS-101X, Qiagen, Valencia, CA, USA). The panel target coverage size is 37.91 kilobases which includes AKT1, ALK, AR, BRAF, CTNNB1, DDR2, EGFR, ERBB2, FGFR3, GNA11, GNAQ, IDH1, IDH2, KIT, KRAS,
MAP2K1, MET, NRAS, PDGFRA, PIK3CA, PTEN, RET, STK11 and TP53. Briefly, 40 nanograms of genomic DNA were used for multiplex polymerase chain reaction (PCR). Then the PCR products were purified using AMPure beads (Beckman Coulter, Brea CA, USA) followed by end repair and adapter ligation using GeneRead Library Prep Kits for Illumina (Qiagen, Valencia, CA, USA). The DNA library was then purified using a GeneRead Size Selection Kit (Qiagen, Valencia, CA, USA) followed by AMPure beads (Beckman Coulter, Brea CA, USA). Quality control of the library construction and library quantification were performed using GeneRead DNAseq library Quant Array following the manufacturer's instructions (Qiagen, Valencia, CA, USA). Then a MiSeq Reagent kit v2 was used to sequence the pair-ends of the target enriched libraries for 300 cycles using the Illumina MiSeq sequencer (Illumina, San Diego, CA, USA). Ten picomolar of DNA libraries with unique indices were pooled with 5.0\% PhiX and loaded per flow cell in each run. Read mapping, variant calling and annotation were performed using QIAGEN's cloud-based GeneRead DNAseq Sequence Variant Analysis Software available at https://geneglobe.qiagen.com/th/analyze. Then variant classification was performed using Ingenuity Variant Analysis $^{\text {TM }}$ (IVA) (Qiagen, CA, USA) and manually reviewed according to the American College of Medical Genetics and Genomics guidelines. ${ }^{12}$ The mutations reported were named based on human genome reference build 37 (GRch37) and RNA reference sequence NM_000546.5. The pathogenic mutations in the patients and their parents, if available, were confirmed using bidirectional direct sequencing.

Baseline characteristics are shown as mean \pm standard deviation, median or percentage, and categorical data as numbers and percentages. The two-tailed independent sample T-test was used to determine differences between the mutation and non-mutation groups. 


\section{Results}

Eight participants were diagnosed with ACC, six girls and two boys, who were enrolled into the study. Three of the eight participants harbored the pathogenic variant of TP53. Participant \#1 was found to have known germlineTP53 mutation [c.1010G>A (p.Arg337His)] (Figure 1A), with parents in which no germline mutation was detected. Participant \#2 had a known mutation [c.916C>T (p.Arg306Ter)] (Figure 1B) and died 10 months after ACC diagnosis following disease progression. Participant \#8 harbored a known mutation [c.743G>A (p.Arg248GIn)];parental samples were unavailable for analysis in this patient. The rest of the participants had wild-type TP53 None of the families of the study participants harboring a germline TP53 mutation had any history of malignancy.
The participants' characteristics are summarized in Table 1. The median ages at onset and at diagnosis were 7.5 months (range 1-62 months) and 25.5 months (range 1067 months), respectively. All participants (100\%) presented with virilization, three (37.5\%) had virilization only and five (62.5\%) also had Cushing. Two participants (participants \#1 and \#2) had pulmonary metastasis at the time of diagnosis. Surgery was performed to completely remove the tumors in all patients, for patients with metastasis, they received adjuvant chemotherapy of doxorubicin, cisplatin and etoposide after tumor removals. One participant (\#8) had recurrence at 12 years after the first tumor was removed and needed a second operation. One wild-type participant (\#3) developed pulmonary recurrence one year after diagnosis and was treated with metastasectomy and

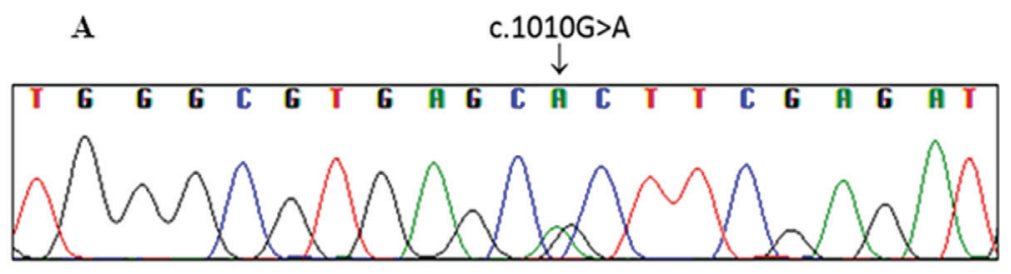

Patient 1

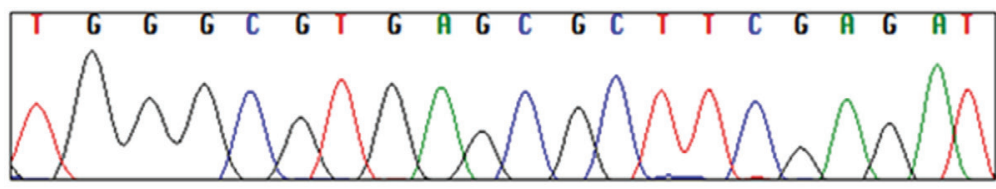

Father

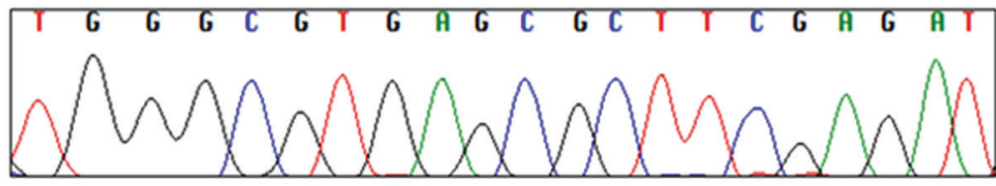

Mother

$\mathbf{B}$

c.916G>A

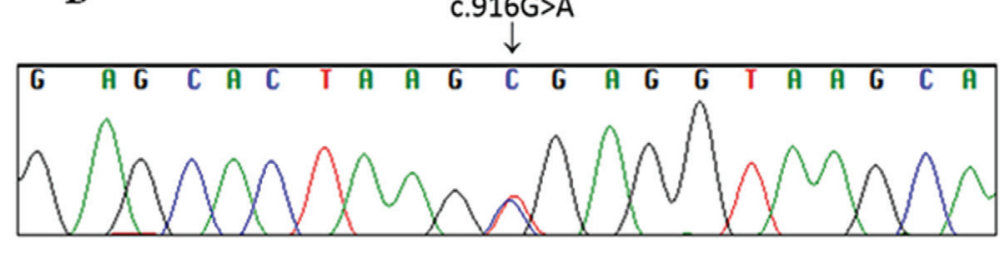

Patient 2

Figure 1 Direct sequencing chromatograms 


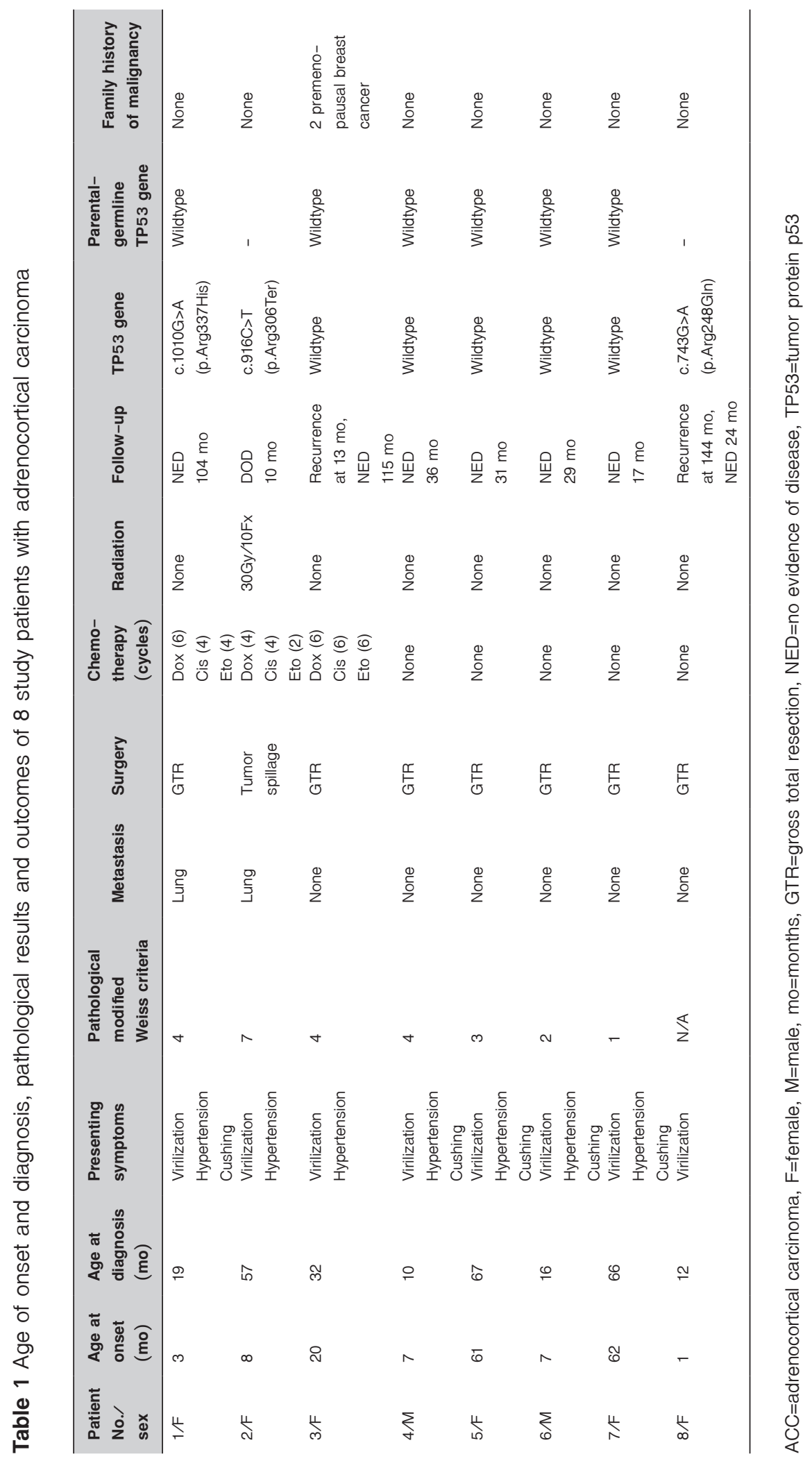


adjuvant chemotherapy. Only one participant (\#2) needed palliative radiotherapy due to intraoperative tumor spillage and disease progression.

The participants were divided into two groups according to the genetic variations, the TP53 mutation group (3 participants) and the wild-type group (5 participants). The median ages at onset of symptoms of ACC in the TP53 mutation and wild-type were 3 months (1-8 months) and 20 months (7-62 months), respectively ( $p$-value=0.097) (Table 2). The median ages at diagnosis of the TP53 mutation and wild type children were 19 and 32 months, respectively $(p-v a l u e=0.652)$. The median durations from onset of symptoms to the diagnosis of pathogenic variants and wild type TP53 were 16 months and 10 months, respectively $(p-v a l u e=0.270)$. The mean weight of the tumors in the participants with the TP53 mutation was $753.0 \pm 703.0$ grams (gm) while in the wild-type group, the mean weight of the tumors was $85.0 \pm 47.9 \mathrm{gm}$. The difference in the mean tumor weights between the two groups was large but not statistically significant ( $p-v a l u e=0.407$ ). Table 2 shows the comparison of the characteristics and clinical symptoms between the participants with the germline TP53 mutation and the wild type group.

Initially at the time of diagnosis, all of the participants had high levels of serum cortisol and loss of diurnal variation regardless of Cushing syndrome features. Only one participant had hypertension and high levels of serum aldosterone. Six participants with hypertension had high levels of serum dehydroepiandrosterone sulfate (DHEAS). The mean serum DHEAS level among the participants with the TP53 mutation and the wild type were 1,569.3 \pm 764.3 and $616.0 \pm 551.0$ micrograms per deciliter $(\mathrm{mcg} / \mathrm{dL})$,

Table 2 Comparison between patients with germline mutation and patients with/wild-type/group

\begin{tabular}{|c|c|c|c|c|}
\hline Baseline characteristic & TP53 mutation & Wildtype & Total & p-value \\
\hline \multirow[t]{3}{*}{ Number, n (\%) } & $3 / 8(38.0)$ & $5 / 8(62.0)$ & $8 / 8$ & \\
\hline & 2/3 (67.0) hotspot & & & \\
\hline & 1/3 (33.0) non-hotspot & & & \\
\hline \multicolumn{5}{|l|}{ Sex } \\
\hline Male, n (\%) & $0 / 3(0.0)$ & $2 / 5(40.0)$ & $2 / 8(25.0)$ & \\
\hline Female, n (\%) & $3 / 3(100)$ & $3 / 5(60.0)$ & $6 / 8(75.0)$ & \\
\hline Median age at the onset, months & 3.0 & 20.0 & 7.5 & 0.097 \\
\hline Median age at diagnosis, months & 19.0 & 32.0 & 25.5 & 0.652 \\
\hline Median duration of time of onset to diagnosis, months & 16.0 & 6.0 & 10.0 & 0.270 \\
\hline \multicolumn{5}{|l|}{ Presenting symptoms, n (\%) } \\
\hline Virilization & $3 / 3(100)$ & $5 / 5(100)$ & $8 / 8(100)$ & \\
\hline Hypertension & $2 / 3(67.0)$ & $5 / 5(100)$ & $7 / 8(88.0)$ & \\
\hline Cushing syndrome & $1 / 3(33.0)$ & $4 / 5(80.0)$ & $5 / 8(63.0)$ & \\
\hline Tumor weight, gram (S.D.) & $753(703.0)^{*}$ & $85(47.9)$ & $276(436.0)$ & 0.407 \\
\hline Metastasis, n (\%) & $2 / 3(67.0)$ & $0 / 5(0.0)$ & $3 / 8(38.0)$ & 0.184 \\
\hline Recurrence, n (\%) & $1 / 3(33.0)$ & $1 / 5(20.0)$ & $2 / 8(25.0)$ & 0.754 \\
\hline Morning serum cortisol (mcg/dL) & $22.5 \pm 14.7$ & $22.0 \pm 11.8$ & $19.8 \pm 12.0$ & 0.962 \\
\hline Morning plasma ACTH (pg/mL) & $14.4 \pm 4.0^{\star}$ & $15.4 \pm 10.5$ & $15.1 \pm 8.8$ & 0.859 \\
\hline Plasma renin $(\mathrm{ng} / \mathrm{mL} / \mathrm{h})$ & $4.5 \pm 1.6^{\star}$ & $8.0 \pm 9.5$ & $7.0 \pm 7.9$ & 0.462 \\
\hline Serum aldosterone (ng/dL) & $10.5 \pm 7.9^{\star}$ & $24.1 \pm 30.6$ & $20.2 \pm 26.0$ & 0.398 \\
\hline Serum DHEAS (mcg/dL) & $1,569.3 \pm 764.3$ & $616.0 \pm 551.1$ & $973.3 \pm 764.2$ & 0.156 \\
\hline
\end{tabular}

TP53=tumor protein p53, ACTH=adrenocorticotropic hormone, mcg/dL=micrograms per deciliter, pg/mL=picograms per milliliter, $\mathrm{ng} / \mathrm{mL} / \mathrm{h}=$ nanograms per milliliter per hour, $\mathrm{ng} / \mathrm{dL}=$ nanograms per deciliter, DHEAs=dehydroepiandrosterone sulfate *data were calculated from 2 patients with available data 
respectively ( $p$-value $=0.156)$ (Table 2$)$. Three months after the tumors were removed, the hormonal values returned to normal in all patients in both groups.

The tumors were completely removed in all participants, but in patient \#2 there was accidental intraoperative tumor spillage. Two of the three participants with the pathogenic variant TP53 had pulmonary metastasis whereas those with the wild type did not have any metastases ( $p$-value=0.184). Tumor recurrence occurred in two participants. One participant who had a mutation had ACC recurrence at 144 months after the first surgery. The other participant, who had a wild-type mutation, had pulmonary recurrence at 13 months after diagnosis. Systemic chemotherapy consisting of doxorubicin, cisplatin and etoposide, were given to three participants with pulmonary tumors. Participant \#2 had a hotspot pathogenic variant. After the tumor was completely removed, participant \#2 received 4 cycles of chemotherapy with palliative radiotherapy. Ten months after the tumor was removed, the disease had progressed and the participant died. The median follow-up time was 31 months (range 10 to 168 months). As of the writing of this report, seven participants are still alive and have not had any recurrences (Figure 2) or second malignancies.

None of the participants with a germline pathogenic variant of TP53 had an history of malignancy in their families (Figure 3) although participant \#3 had several female members who had had pre-menopausal breast cancer. We suspect that this could be from another gene mutation.

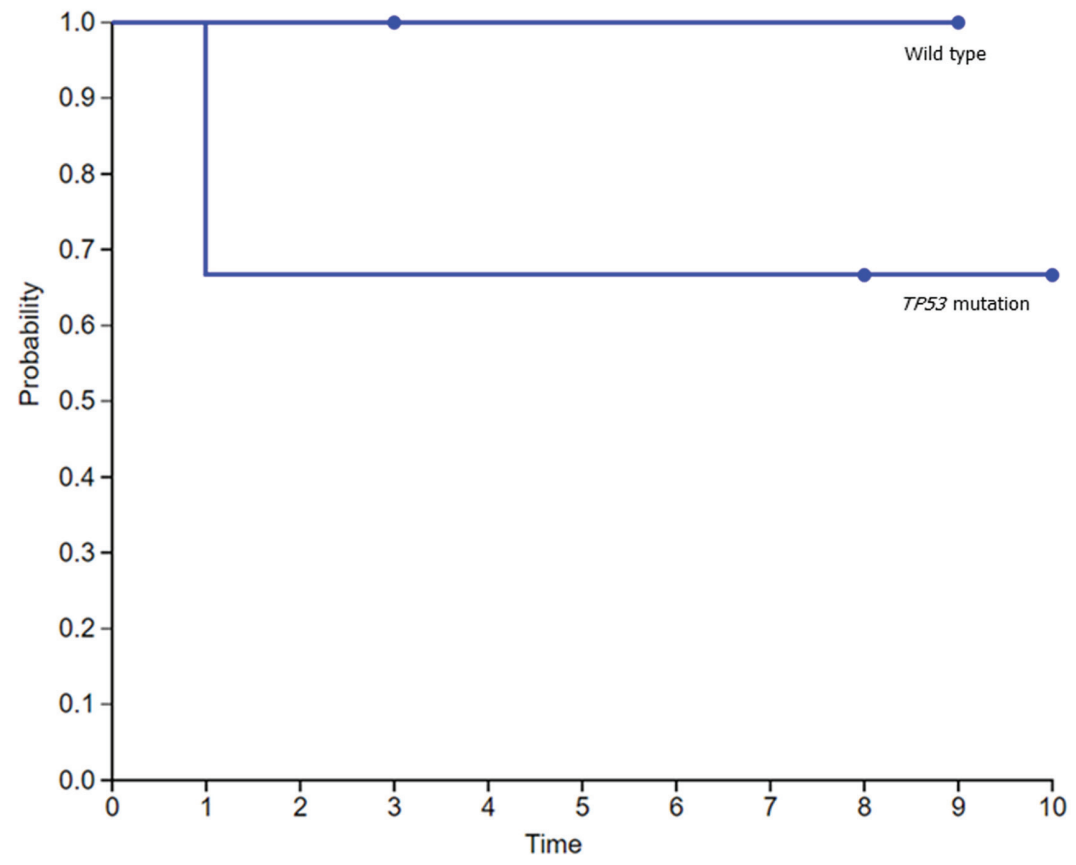

Figure 2 Accumulative probability of surviving 


\section{A. Patient 1}

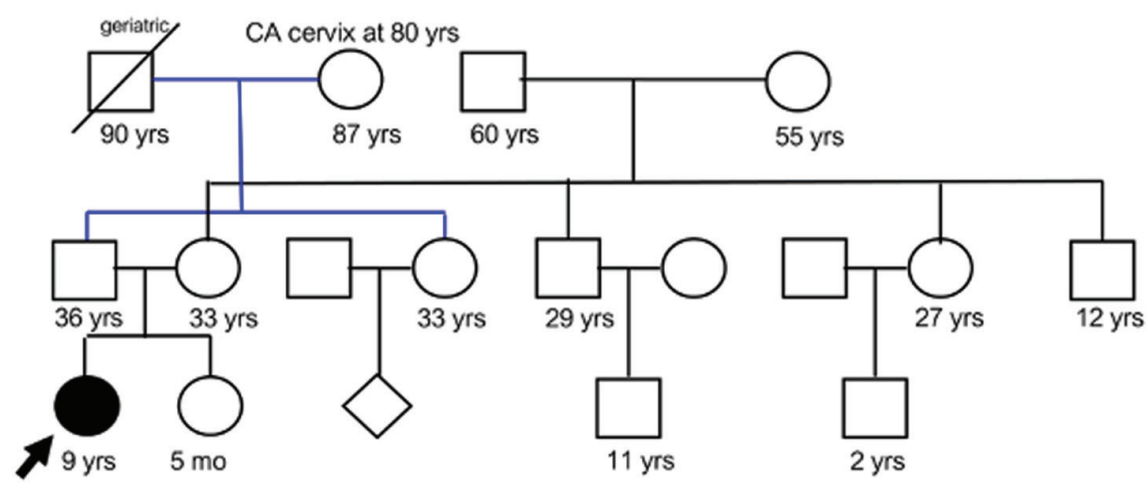

\section{B. Patient 2}

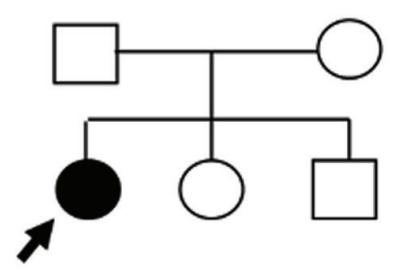

C. Patient 3

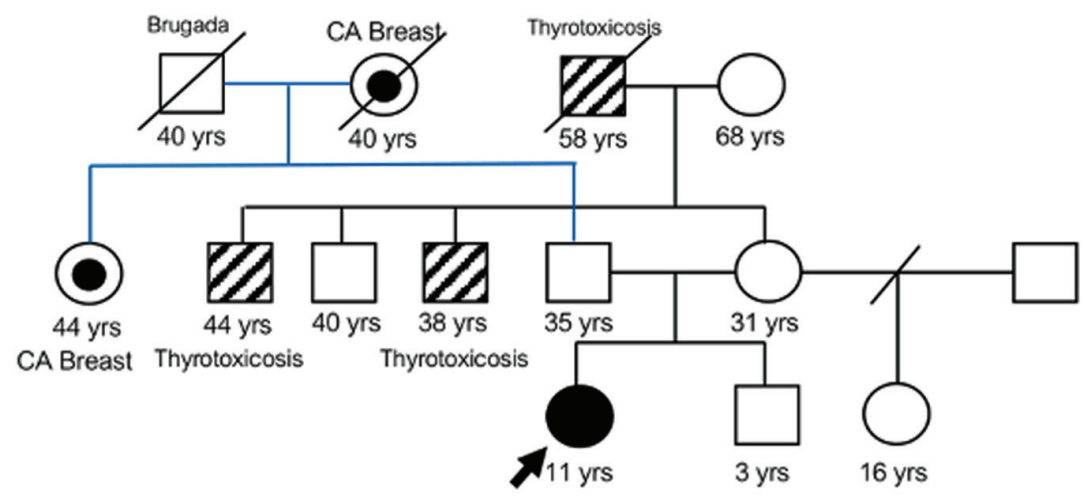

$\mathrm{CA}=$ carcinoma

Figure 3 Family pedigrees 


\section{Discussion}

To our knowledge, this is the first study of germline pathogenic variants of TP53 in Thai children with ACC. Given its rarity, this study had recruited a total of eight participants with ACC over a 10-year period.

Most of our participants had shown excess adrenal hormones during their early childhood. This finding is consistent with a previous report. ${ }^{1}$ All of them presented with virilization, while only five of them had Cushing appearance. The hormonal profiles all showed high levels of serum DHEAS which was consistent with the virilization presented in all patients, and high levels of serum cortisol regardless of Cushing appearance. The elevated levels of DHEAS is a sensitive marker to detect ACC. ${ }^{1}$ In this study, we found only one participant with high level of serum aldosterone. A prior study found that primary hyperaldosteronism was rare in ACC. ${ }^{1}$ We propose that the elevated blood pressure in children with ACC could result from either hypersecretion of cortisol or aldosterone and other mineralocorticoids produced by adrenocortical tumors. ${ }^{13}$ Considering the overall outcomes, almost all patients survived but two participants had recurrence. Only one participant (12.0\%) died of progressive disease. This participant had a large tumor size (1,250 gm), intraoperative tumor spillage and distant metastasis which were poor prognostic factors. ${ }^{14,15}$ In our study, metastasis was found only in the lungs. However, earlier studies from the USA and Italy reported that the most common metastasis site in ACC was the liver. ${ }^{15}$ In our study, the survival probability was high which was in line with a previous report from the USA. ${ }^{2}$ In our study, three participants with a pulmonary tumor received adjuvant cytotoxic chemotherapy of doxorubicin, cisplatin and etoposide. The overall prognosis of childhood ACC is good when the metastatic tumor is completely removed and the child is treated with adjuvant chemotherapy. However, this is not true for patients with distinct high-risk ACC features, namely large tumor size and tumor spillage, as in one of our participants with a fatal outcome. ${ }^{14}$
We detected germline TP53 pathogenic variants in three participants out of eight participants (38.0\%). The frequency of these pathogenic variants was low compared to previous reports from Latin America and Malaysia. In Europe, the frequency of these pathogenic variants was $7.3 \%$ which was much lower compared to our study. ${ }^{14}$ We propose that these variations may be due to different ethnicities and the small number of cases in our study. We searched for publications pertaining to the prevalence of germline TP53 mutations in children with ACC in PubMed. We excluded studies on $\mathrm{R} 337 \mathrm{H}$ from Brazil and studies conducted in adults. We used the terms "TP53 and ACC" as keywords and also extended our review to the references of these publications. Our findings are summarized in Table 3.

The pathogenic variants detected in this study were all known variants in childhood ACC. One of the participants in our study had a germline TP53 mutation and no family history of premenopausal breast cancer, osteosarcoma or other soft tissue sarcoma. For the other participants, we could not prove whether the mutation was inherited or de novo because we did not have samples from the participants' parents.

Based on the results of our study, checking the hotspot TP53 mutation on exons 5-8 was not sufficient because one of our participants had this mutation located on exon 10. This finding is consistent with a previous study which found that only $5.9 \%$ of patients with a germline TP53 mutation had a hotspot mutation. ${ }^{16}$ Therefore, we recommend that the whole TP53 gene should be sequenced to detect all mutations of ACC.

When we compared the clinicopathologic features of patients with the TP53 mutation and the wild-type group, we found that there were significant differences between the two groups. The patients with the TP53 mutations had earlier onset of symptoms, larger tumor size, pulmonary metastasis and higher levels of serum DHEAS compared to those patients with the wild type. One participant with 
a TP53 mutation experienced tumor recurrence more than 10 years after remission. One factor that can affect the treatment outcome is the duration of time between onset and diagnosis. One study reported that delays in the diagnosis of ACC in patients with pathogenic variants resulted in poorer treatment outcomes regardless of other pathological features such as breast cancer with TP53 mutation. ${ }^{17}$ Besides poorer prognosis, the disease tended to progress more quickly in patients with TP53 mutations than patients with wild type TP53. However, these findings were not significantly different between the two study groups, and studies with larger groups of patients are required for more conclusive results. In this study, other changes in the adrenal cortex hormones were not different between participants with TP53 mutations and wild-type TP53.

In our study, a history of familial malignancy alone could not distinguish between participants with the TP53 mutation or wild-type TP53. Firstly, none of the participants who had the TP53 mutation reported any familial cancers. Since ACC with the TP53 mutation tended to be more progressive, it would be beneficial for the patient to be able to detect a TP53 mutation. This would help physicians select the most appropriate treatment for the patient, improve the monitoring system for the disease after complete removal of the tumor, and provide good cancer surveillance for other family members. Thus, we emphasize that all patients

Table 3 Reviewed publications regarding prevalence of germline TP53 mutations in childhood adrenocortical carcinoma

\begin{tabular}{|c|c|c|c|c|c|c|}
\hline No. & $\begin{array}{l}\text { Author }(\mathbf{s}) \\
\text { (publication year) }\end{array}$ & Country(ies) & $\begin{array}{l}\text { Median age at } \\
\text { diagnosis } \\
\text { (years, range) }\end{array}$ & $\begin{array}{l}\text { No. } \\
\text { of cases } \\
(n)\end{array}$ & $\begin{array}{l}\text { No. of germline } \\
\text { TP53 mutations } \\
(\mathrm{n}, \%)\end{array}$ & $\begin{array}{l}\text { Method of mutation } \\
\text { analysis }\end{array}$ \\
\hline 1 & $\begin{array}{l}\text { Josephine Wagner, et al. } \\
(1994)^{7}\end{array}$ & Canada, USA & $4.8^{*}(1.0-17.0)$ & 6 & $3(50.0)$ & $\begin{array}{l}\text { Single strand conformational } \\
\text { polymorphism }\end{array}$ \\
\hline 2 & $\begin{array}{l}\text { Jennifer M. Varley, et al. } \\
(1999)^{22}\end{array}$ & England & $3.5(1.0-5.0)$ & 11 & $9(82.8)$ & $\begin{array}{l}\text { Single strand conformational } \\
\text { polymorphism }\end{array}$ \\
\hline 3 & $\begin{array}{l}\text { Kelly D. Gonzalez, et al. } \\
(2009)^{66}\end{array}$ & Canada, USA & $9.7^{*}(0.5-38.0)$ & 21 & $14(66.7)$ & Direct sequencing \\
\hline 4 & $\begin{array}{l}\text { Emilia M. Pinto, et al. } \\
(2011)^{8}\end{array}$ & Brazil, USA & N/A $(0.0-19.0)^{\mp}$ & 48 & $36(75.0)$ & N/A (exon 2 through 11) \\
\hline 5 & $\begin{array}{l}\text { Susanne Magnusson, } \\
\text { et al. }(2012)^{23}\end{array}$ & Sweden & $6.1(3.5-14.9)$ & 3 & $1(33.3)$ & $\begin{array}{l}\text { multiplex ligation-dependent } \\
\text { probe amplification }\end{array}$ \\
\hline 6 & $\begin{array}{l}\text { Siew Shean Choong, } \\
\text { et al. }(2012)^{9}\end{array}$ & Malaysia & N/A $(3.0-10.0)^{\mp}$ & 4 & $3(75.0)$ & BioEdit Sequencing \\
\hline 7 & $\begin{array}{l}\text { Jonathan D. Wasserman, } \\
\text { et al. }(2015)^{16}\end{array}$ & Canada, USA & N/A & 68 & $34(50.0)$ & $\begin{array}{l}\text { BigDye direct Sanger } \\
\text { sequencing }\end{array}$ \\
\hline 8 & $\begin{array}{l}\text { Giovanni Cecchetto, et al. } \\
(2016)^{14}\end{array}$ & $\begin{array}{l}\text { Italy, Poland, } \\
\text { France, } \\
\text { Germany }\end{array}$ & $8.1(2.7-14.0)$ & 82 & $6(7.3)$ & N/A \\
\hline 9 & $\begin{array}{l}\text { Mariette Renaux-Petel, } \\
\text { et al. }(2017)^{24}\end{array}$ & France & N/A & 97 & $47(48.5)$ & $\begin{array}{l}\text { direct DNA sequencing ( } 45 \\
\text { cases), NGS (additional } 2 \\
\text { cases) }\end{array}$ \\
\hline
\end{tabular}

USA=United States of America, DNA=deoxyribonucleic acid, NGS=next generation sequencing, TP53=tumor protein p53, N/A=not applicable *mean age, ${ }^{\ddagger}$ Only range available 
with ACC should be tested for the presence of the TP53 mutation regardless of family history.

Secondly, TP53 mutations can cause both ACC and premenopausal breast cancer. In our study there were multiple premenopausal breast cancers in the family of one participant who did not have TP53 mutation but had tumor recurrence. We propose that this family might have other mutations which can cause both ACC and hereditary breast cancers, namely the BRCA1/BRCA2 and CHEK2 mutations. ${ }^{18-21}$ However, these mutations are rare in ACC. Further gene analysis in this family is required.

\section{Conclusion}

We successfully identified three different germline TP53 mutations in participants with ACC. The disease was more progressive among participants with a TP53 mutation in terms of tumor recurrence and metastasis. Also, these participants all had pulmonary metastasis. Direct sequencing of only the hotspot region is not sufficient. It is recommended that the full TP53 gene or the whole exome be sequenced. Participants with a TP53 mutation had poorer prognosis and earlier onset of symptoms. Detection of this mutation would help the patients and their relatives to detect malignancies during the asymptomatic phase.

\section{Funding sources}

This study was supported by the Ratchadapiseksompotch Fund, Faculty of Medicine, Chulalongkorn University, grant number RA61/052.

\section{Conflict of interest}

No

\section{References}

1. Ribeiro RC, Michalkiewicz EL, Figueiredo BC, DeLacerda L, Sandrini F, Pianovsky MD, et al. Adrenocortical tumors in children. Braz J Med Res 2000;33:1225-34.
2. Mahendraraj K, Lau CSM, Sindhu K, Chamberlain RS. Adrenocortical carcinoma in adults and children: a population-based outcomes study involving 1,623 patients from the surveillance, epidemiology, and end result (SEER) database (1973-2012). Clin Surg 2016;1:1-7.

3. Abiven G, Coste J, Groussin L, Anract P, Tissier F, Legmann P, et al. Clinical and biological features in the prognosis of adrenocortical cancer: poor outcome of cortisol secreting tumors in a series of 202 consecutive patients. J Clin Endocrionol Metab 2006;91:2650-5.

4. Sandrini R, Ribeiro RC, DeLacerda L. Extensive personal experience childhood adrenocortical tumor. J Clin Endocrinol Metab 1997;82:2027-31.

5. Ribeiro RC, Pinto EM, Zambetti GP. Familial predisposition to adrenocortical tumors: clinical and biological features and management strategies. Best Pract Res Clin Endocrinol Metab 2010;24:477-90.

6. Gonzalez KD, Noltner KA, Buzin CH, Gu D, Wen-Fong CY, Nguyen VQ, et al. Beyond Li Fraumeni syndrome: clinical characteristics of families with p53 germline mutations. J Clin Oncol 2009;27:1250-6.

7. Wagner J, Portwine C, Rabin K, Leclerc J, Narod SA, Malkin D. High frequency of germline p53 mutations in childhood adrenocortical cancer. J Natl Cancer Inst 1994;86:1707-10.

8. Pinto EM, Ribeiro RC, Figueiredo BC, Zambetti GP. Tp53associated pediatric malignancies. Genes Cancer 2011;2:48590.

9. Choong SS, Latiff ZA, Mohamed M, Lim LLW, Chen KS, Vengidasan L, et al. Childhood adrenocortical carcinoma as a sentinel cancer for detecting families with germline TP53 mutations. Clin Genet 2012;82:564-8.

10. Herrmann LJ, Heinze B, Fassnacht M, Willenberg HS, Quinkler M, Reisch N, Zink M, Allolio B, Hahner S. TP53 germline mutations in adult patients with adrenocortical carcinoma. J Clin Endocrinol Metab 2012;97:E476-85.

11. Salmon A, Amikam D, Sodha N, Davidson S, Basel-Vanagaite L, Eeles RA, et al. Rapid development of post-radiotherapy sarcoma and breast cancer in a patient with a novel germline “De-Novo” TP53 mutation. Clin Oncol 2007;19:490-3.

12. Richard S, Aziz N, Bale S, Bick D, Das S, Gastier-Foster J, et al. Standards and guidelines for the interpretation of sequence variants: a joint consensus recommendation of the 
American College of Medical Genetics and Genomics and the Association for Molecular Pathology. Genet Med 2015;17: 405-24.

13. Egoshi K, Masai M, Nagao K, Ito H. 11-Deoxycorticosteroneproducing adrenocortical carcinoma. Urol Int 1999;251-3.

14. Cecchetto G, Bisogno G, Schneider D, Brecht I, Ganarin A, Bien $E$, et al. Outcome and prognostic factors in high-risk childhood adrenocortical carcinomas: a report from the European Cooperative Study Group on Pediatric Rare Tumors (EXPeRT). Pediatr Blood Cancer 2017;64. doi: 10.1002/pbc. 26368.

15. Gupta N, Rivera M, Novotny P, Rodriguea V, Bancos I, Lteif A. Adrenocortical carcinoma in children: a clinicopathological analysis of 41 Patients at the Mayo Clinic from 1950 to 2017. Horm Res Paediatr 2018;90:8-18.

16. Wasserman JD, Novokmet A, Eichler-Jonsson C, Ribeiro RC, Rodriguez-Galindo C, Zambetti GP, et al. Prevalence and functional consequence of TP53 mutations in pediatric adrenocortical carcinoma: a children's oncology group study. J Clin Oncol 2015;33:602-9.

17. Petitjean A, Achatz MIW, Borresen-Dale AL, Hainaut P, Olivier M. TP53 mutations in human cancers: functional selection and impact on cancer prognosis and outcomes. Oncogene 2007;26:2157-65.

18. Couch FJ, Shimelis H, Hu C, Hart SN, Lilyquist J, Feng B, et al. Associations between cancer predisposition testing panel genes and breast cancer. JAMA Oncol 2018;3:1190-6.
19. Ghorayeb N El, Grunenwald S, Nolet S, Primeau V, Côté S, Maugard CM, et al. First case report of an adrenocortical carcinoma caused by a BRCA2 mutation. Med 2016;95. doi: 10.1097/MD.0000000000004756.

20. Kim H, Doo DC, Choi H. Frequency of pathogenic germline mutation in CHEK2, PALB2, MRE11, and RAD50 in patients at high risk for hereditary breast cancer. Breast Cancer Res Treat 2017;161:95-102.

21. Xie C, Tanakchi S, Raygada M, Davis JL. Case report of an adrenocortical carcinoma associated with germline CHEK2 mutation. J Endocr Soc 2019;3:284-90.

22. Varley JM, McGown G, Thorncroft M, James LA, Margison GP, Forster G, et al. Are there low-penetrance TP53 Alleles? evidence from childhood adrenocortical tumors. Am J Hum Genet 1999;65:995-1006.

23. Magnusson S, Gisselsson D, Wiebe T, Kristoffersson U, Borg $\mathrm{A}$, Olsson $\mathrm{H}$. Prevalence of germline TP53 mutations and history of Li-Fraumeni syndrome in families with childhood adrenocortical tumors, choroid plexus tumors, and rhabdomyosarcoma: A population-based survey. Pediatr Blood Cancer 2012;59: 846-53.

24. Renaux-petel M, Charbonnier F, Théry J, Fermey P, Lienard G, Bou J, et al. Contribution of de novo and mosaic TP53 mutations to Li-Fraumeni syndrome. J Med Genet 2018;55:173-80. 